\title{
A Lower Bound on the Greedy Weights of Product Codes and Projective Multisets
}

\author{
Hans Georg Schaathun ${ }^{1}$ \\ Department of Informatics \\ University of Bergen \\ Høyteknologisenteret \\ N-5020 Bergen, Norway \\ e-mail: georg@ii.uib.no
}

\begin{abstract}
We present the relation between product codes and projective multisets, and give a lower bound on the greedy weights of product codes.
\end{abstract}

\section{GREEDY WEIGHTS}

The weight hierarchy has received a lot of attention after Victor Wei's paper [5]. Let $C$ be an $[n, k]$ linear code. The support of a codeword $c \in C$ is the set $\chi(c)$ of non-zero coordinate positions. For a subcode $D \subseteq C$ we write $\chi(D)=\bigcup_{c \in D} \chi(c)$. The support size \# $\chi(D)$ is called the weight of $D$. The weight hierarchy of $C$ is the sequence $\left(d_{1}, d_{2}, \ldots, d_{k}\right)$, where $d_{r}$ is the least weight of an $r$-dimensional subcode.

When the code is applied on the Wire-Tap Channel of Type II, $d_{i}$ is the number of channel symbols needed to acquire $i$ information symbols.

The greedy weights are inspired by the following problem: Suppose the adversary uses a greedy approach. First he reads $d_{1}$ symbols to get one information symbol. After having got $i-1$ information symbol, his top priority is to get the $i$-th bit as soon as possible. The total number of bits required to get $i$ information symbols by this greedy approach is called the $i$-th greedy weight and denoted $g_{i}$; and $g_{2}$ has been studied in $[1,2]$.

The number $g_{i}$ may equivalently be defined recursively from the code. Let a greedy 1-subcode be a subcode of dimension 1 and weight $g_{1}=d_{1}$. Let a greedy $i$-subcode be a subcode of dimension $i$ and as small weight as possible under the constraint that it contain a greedy $(i-1)$-subcode. Then $g_{i}$ is the weight of a greedy $i$-subcode.

\section{Projective Multisets}

A $q$-ary $[n, k]$ code $C$ is determined completely by its generator matrix $G$. The rows of $G$ constitute a basis for $C$. The columns of $G$ make the projective multiset $\gamma_{C}$ corresponding to $C$. A projective multiset is a collection of projective points, not necessarily distinct, and it is often written as a map

$$
\gamma_{C}: \mathrm{PG}(k-1, q) \rightarrow\{0,1,2, \ldots\},
$$

where the value $\gamma_{C}(x)$ of $x$ is the number of times $x$ occurs in the collection. The value of a set $S$ as $\gamma_{C}(S)=\sum_{x \in S} \gamma_{C}(x)$.

It is well known that there is a one-to-one correspondence between subcodes $D \subseteq C$ of dimension $i$ and subspaces $\Pi \subseteq$ $\mathrm{PG}(k-1, q)$ of codimension $i$, such that \# $\chi(D)=n-\gamma_{C}(\Pi)$.

The subspaces corresponding to greedy subcodes are called greedy subspaces. We can equivalently define a greedy subspace of codimension 1 to be a hyperplane of value $n-d_{1}=$

${ }^{1}$ Part of this work was done as a visiting researcher at Ecole Nationale Supérieure de Télécommunications in Paris, France, with support from The Norwegian Research Council under Grant $138654 / 410$ $n-g_{1}$. Then we define a greedy subspace of codimension $i$ to be a subspace of maximum value under the constraint that it be contained in a greedy subspace of codimension $i-1$. The value of a greedy subspace of codimension $i$ is $n-g_{i}$.

\section{Product Codes}

The product code $C=A \otimes B$ is the tensor product of $A$ and $B$ as vector spaces. In [3] we saw that $\gamma_{C}$ is obtained by applying the Segre embedding on $\gamma_{A}$ and $\gamma_{B}$. As a consequence we found a lower bound on the weight hierarchy of product codes and proved the Wei-Yang conjecture. These results have been generalised in [4]. Here we use the same technique to bound the greedy weights. The bound we find is quite similar to the existing one on the weight hierarchy, but the proof requires more care.

Define

$$
\mathcal{M}_{t}:=\left\{\mathbf{i}=\left(i_{1}, i_{2}, \ldots, i_{t-1}\right) \mid 1 \leq i_{j} \leq k_{j}, 1 \leq j<t\right\} .
$$

Definition 1 Let $\pi$ be a map $\mathcal{M}_{t} \rightarrow\left\{0,1, \ldots, k_{t}\right\}$ given by $\mathbf{i} \mapsto t_{\mathbf{i}}$. We call $\pi a\left(k_{1}, k_{2}, \ldots, k_{t}\right)$-partition of $r$ if

1. $\sum_{\mathrm{i} \in \mathcal{M}_{t}} t_{\mathrm{i}}=r$.

2. $\pi$ is a decreasing function in each coordinate, i.e. $t_{i_{1}, \ldots, i_{j}, \ldots, i_{t-1}} \leq t_{i_{1}, \ldots, i_{j}-1, \ldots, i_{t-1}}$ for $j=1, \ldots, t-1$ and $1<i_{j}$.

The set of all $\left(k_{1}, k_{2}, \ldots, k_{t}\right)$-partitions of $r$ is denoted by $\mathcal{P}\left(k_{1}, \ldots, k_{t} ; r\right)$.

Theorem 1 If $C_{1}, C_{2}, \ldots, C_{t}$ are linear codes, then

$$
g_{r}\left(C_{1} \otimes \ldots \otimes C_{t}\right) \geq \min \left\{\nabla_{E}(\pi) \mid \pi \in \mathcal{P}\left(k_{1}, \ldots, k_{t} ; r\right)\right\},
$$

where

$$
\nabla_{E}(\pi):=\sum_{\mathbf{i} \in \mathcal{M}_{t}} \prod_{j=1}^{t-1}\left(g_{i_{j}}\left(C_{j}\right)-g_{i_{j}-1}\left(C_{j}\right)\right) g_{\pi(\mathbf{i})}\left(C_{t}\right) .
$$

It was proved in [2] that this bound holds with equality for $r=2$.

\section{REFERENCES}

[1] Wende Chen and Torleiv Kløve. On the second greedy weight for linear codes of dimension 3. To appear in Discrete Mathematics.

[2] Gérard D. Cohen, Sylvia B. Encheva, and Gilles Zémor. Antichain codes. Des. Codes Cryptogr., 18(1-3):71-80, 1999.

[3] Hans Georg Schaathun. The weight hierarchy of product codes. IEEE Trans. Inform. Theory, 46(7):2648-2651, November 2000.

[4] Hans Georg Schaathun and Wolfgang Willems. A lower bound for the weight hierarchy of product codes and projective multisets. In Proc. IEEE Intern. Symp. Inform. Theory, June 2001.

[5] Victor $\mathbb{K}$. Wei. Generalized Hamming weights for linear codes. IEEE Trans. Inform. Theory, 37(5):1412-1418, 1991. 